\title{
La peste en Europe et la peinture italienne : relecture du Supplice de Marsyas de Titien
}

La peste in Europa e la pittura italiana: rilettura del Supplizio di Marsia di Tiziano

The Plague in Europe and Italian Painting: Rereading Titian's Supplice of Marsyas

\section{Silvie Bernier}

\section{OpenEdition Journals}

Édition électronique

URL : http://journals.openedition.org/cei/7431

DOI : 10.4000/cei.7431

ISSN : 2260-779X

Éditeur

UGA Éditions/Université Grenoble Alpes

Édition imprimée

ISBN : 978-2-37747-225-3

ISSN : $1770-9571$

Référence électronique

Silvie Bernier, «La peste en Europe et la peinture italienne : relecture du Supplice de Marsyas de Titien », Cahiers d'études italiennes [En ligne], 31 | 2020, mis en ligne le 06 octobre 2020, consulté le 26 mars 2021. URL : http://journals.openedition.org/cei/7431 ; DOI : https://doi.org/10.4000/cei.7431

Ce document a été généré automatiquement le 26 mars 2021.

(c) ELLUG 


\section{La peste en Europe et la peinture italienne : relecture du Supplice de Marsyas de Titien}

La peste in Europa e la pittura italiana: rilettura del Supplizio di Marsia di Tiziano

The Plague in Europe and Italian Painting: Rereading Titian's Supplice of

Marsyas

Silvie Bernier

1 Arrivée d'Extrême-Orient, en passant par l'Iran et la Mésopotamie avant d'atteindre les ports de Sicile puis de Marseille, la peste noire s'est propagée à travers l'Europe dès la moitié du XIV siècle ${ }^{1}$. L'épidémie trouve un terreau fertile dans les agglomérations urbaines où la concentration de la population favorise la contamination. De 1345 à 1730, des foyers d'infection font des ravages dans les villes de Naples, Rome, Florence, Paris, Bruxelles, Londres, Vienne, notamment ${ }^{2}$. L'Italie, première frappée sur le continent, met en place dès la deuxième moitié $d u x^{e}$ siècle une série de mesures d'hygiène qui servira de modèle aux autres pays d'Europe ${ }^{3}$. Plaque tournante des échanges commerciaux entre l'Orient et l'Occident dès le Moyen Âge, la ville de Venise est durement touchée par l'épidémie. Entre 1575 et 1577, au moment où Titien peint Le supplice de Marsyas (fig. 1), Venise subit la pire éclosion de son histoire qui tue en deux ans le tiers de la population de la ville ${ }^{4}$ et emporte avec elle le peintre déjà vieux et quelques mois plus tard son fils et assistant Octavio $^{5}$. Francesco Sansovino, fils de Jacopo, décrit l'horreur et l'effroi qui règnent dans la ville où les morts s'accumulent et transforment les lieux publics en véritables cimetières: "[...] habbiamo veduto per la horrenda peste di questo anno, ripieno [l'édifice Lazaretto Vecchio] di otto mila persone infettate, con gran danno \& dolore dell'afflitta città [... $]^{6} . »$ 
Figure 1. - Tiziano Vecellio, Le supplice de Marsyas, circa 1576.

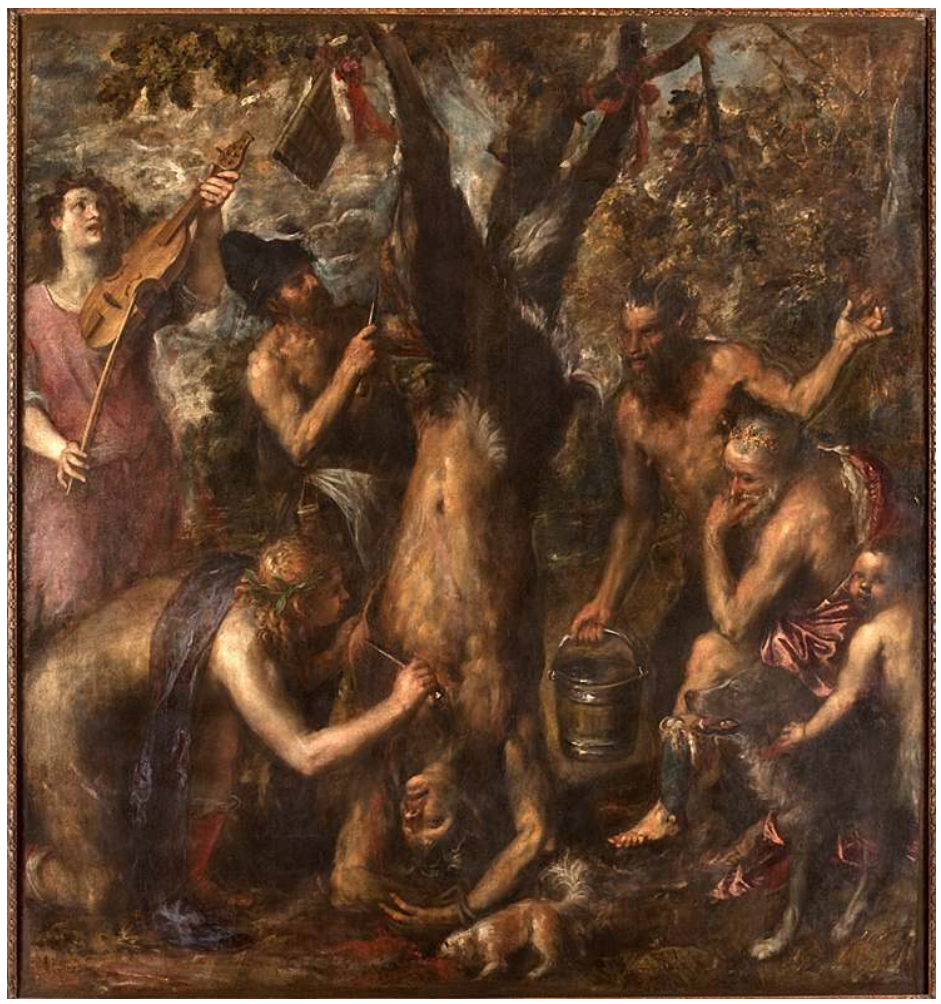

Huile sur toile, $212 \times 207 \mathrm{~cm}$.

Musée national de Kroměřiž, République tchèque.

2 Cet épisode tragique de l'histoire de Venise - et de la biographie de l'artiste - me parait indissociable du tableau qu'Erwin Panofsky jugeait si terrible qu'il refusait d'en attribuer la paternité à Titien ${ }^{7}$. Redécouvert en 1924 dans un obscur château de Kroměříž en République tchèque ${ }^{8}$, le tableau reste muet sur ses conditions de production'. On ignore s'il est le résultat d'une commande, mais le sujet mythologique laisse supposer un commanditaire privé. On sait uniquement qu'il faisait partie de la vaste collection du conte d'Arundel Thomas Howard à sa mort en 1646 et que celui-ci l'aurait acquis d'une riche famille vénitienne ${ }^{10}$. Même l'état d'achèvement du tableau pose problème. Si William R. Rearick assure qu'il se trouvait parmi les œuvres inachevées retrouvées dans le studio de Titien au moment de sa mort en $1576^{11}$, pour David Rosand, la signature en bas à droite de la toile confirme que l'artiste en avait approuvé l'état définitif ${ }^{12}$. Des radiographies révèlent pourtant des ajouts au tableau original qui ne seraient pas de la main de Titien : l'enfant au chien à droite et l'ange jouant de la viole à gauche. Ces détails, qui n'en sont pas, n'apparaissent pas non plus sur une copie anonyme contemporaine du tableau. Ces nouveaux «éléments de preuve » conduisent Augusto Gentili à qualifier Le supplice de Marsyas de « chef d'œuvre inachevé-presque achevé ${ }^{13}$ ».

3 À la suite de l'exposition Genius of Venice tenue en 1983 à la Royal Academy of Arts de Londres, Le supplice de Marsyas éveille l'intérêt des historiens d'art et donne lieu à de nombreuses interprétations iconographiques ${ }^{14}$. Au traitement pour le moins cavalier réservé à l'œuvre par Panofsky ${ }^{15}$, des auteurs opposent une lecture symbolique qui l'inscrit dans des débats sur la peinture, la religion, l'identité, le pouvoir ${ }^{16}$. L'œuvre tardive de Titien, faut-il le préciser, ne présente aucun lien apparent avec l'épidémie de 
peste contrairement à son Saint-Sébastien (1520), une figure attestée dans la religion catholique du combat contre la peste ${ }^{17}$. Le supplice de Marsyas illustre la fable tirée des Métamorphoses d'Ovide où le satyre Marsyas, à la suite d'un défi musical perdu contre le dieu Apollon, se voit puni et dépouillé de sa peau. Le caractère cru du traitement de la fable d'Ovide et la gestuelle expressive du peintre sont pour certains le signe d'une véritable modernité, comparable au Guernica de Picasso ${ }^{18}$.

Susan Sontag, dans son essai Devant la douleur des autres ${ }^{19}$, a avoué son incapacité à regarder le tableau de Titien. Cette vision d'horreur est pour elle insoutenable. La scène cadrée de près et abordée frontalement est, dans les faits, provocante au sens où elle provoque une réaction à la fois de stupeur et de fascination. Stupeur devant l'extrême violence représentée - ce que Panofsky a qualifié de «brutalité gratuite » (gratuitous brutality $)^{20}$ - et fascination devant la sensualité et la vibration de la matière picturale. Cette tension est exacerbée par une série d'antinomies, qu'ont signalées les historiens d'art, entre le divin et le mortel, l'humain et l'animal, l'esprit et le corps, le vivant et le mort, l'apollonien et le dionysiaque ${ }^{21}$.

Pourtant, peu d'auteurs ont rapproché le contexte sociohistorique de production du tableau, cette épidémie de peste ravageuse, et la scène représentée par Titien à travers le mythe de Marsyas. Face à l'horreur illustrée dans le tableau - qui continue d'agir sur le spectateur d'aujourd'hui si on se fie au commentaire de Sontag - comment faire abstraction d'une des plus grandes peurs de l'histoire du Moyen Âge et des débuts de l'époque moderne, qui a profondément imprégné l'imaginaire collectif ${ }^{22}$ ?

Dans un article sur l'imaginaire à la Renaissance, François Azouvi a tenté un rapprochement entre «trois figures du malheur » propres à cette époque, c'est-à-dire la peste, la mélancolie et le diable, qui ont en commun un trait qui intéresse le peintre et l'historien de l'art: la couleur noire ${ }^{23}$. Bien qu'Azouvi ne traite ni d'art ni de peinture, le lien qu'il propose entre ces « objets ", qui font référence au contenu et à la forme, trouve une résonnance inattendue dans Le supplice de Marsyas de Titien. La mort noire comme l'appelaient ses contemporains s'introduit dans le tableau et ajoute une nouvelle couche d'interprétation à cette fable mythologique maintes fois représentée et commentée. L'identité des personnages, Apollon, Marsyas, le roi Midas, mais aussi le sens des procédés picturaux, s'en trouvent renouvelés. Le contexte socio-historique et l'imaginaire de la peste situent autrement l'œuvre dans le langage et la pratique de la médecine, de la théologie et de la peinture à la Renaissance, dont elle semble précisément marquer la fin.

\section{La peste noire et les pizzigamorti}

7 Ambroise Paré (1510-1590), premier chirurgien du roi de France et considéré comme le père de la chirurgie moderne ${ }^{24}$, a décrit les ravages de la peste, dans son Traicté de la Peste, de la petite Verolle \& Rougeolle: "C'est une maladie furieuse, qui court généralement sur tous les hommes, ou sur les autres bêtes, contagieuse, cruelle et pernicieuse $[. . .]^{25}$. $\gg$ La peste noire ou peste bubonique se caractérise par le gonflement de ganglions lymphatiques (les «bubons ») qui soulèvent la peau au cou, aux aisselles et à l'aine. Des hémorragies sous-cutanées provoquent des ecchymoses ${ }^{26}$ sombres si bien que la langue, les lèvres et les ongles du pestiféré deviennent noirs. Au dernier stade de la maladie, c'est tout le corps qui noircit ${ }^{27}$. D'autres symptômes tels de fortes fièvres, 
des nausées, des vomissements, de violents maux de tête complètent ce sombre portrait clinique $^{28}$.

Dans le tableau de Titien, le corps de Marsyas est pleinement exposé comme le serait celui d'un malade ausculté par un médecin. Son corps, les jambes écartées et les bras pendants, forme une croix qui rappelle celles qu'on peignait sur la façade des maisons aux temps de la peste pour condamner les habitations infectées. Le bas du corps du satyre est déjà noir puisque recouvert d'une fourrure sombre. Giulio Romano, dans un dessin à la plume sur le même sujet, préparatoire à la fresque ${ }^{29}$ aujourd'hui endommagée du Palazzo Te ${ }^{30}$ (fig. 2), avait représenté la nature animale de Marsyas uniquement par ses sabots. Titien, lui, accentue l'hybridité du satyre en peignant des pattes et un bas-ventre de bouc qui l'apparente au dieu $\operatorname{Pan}^{31}$, un autre personnage mythologique mis en scène par Ovide qui entra en compétition avec Apollon ${ }^{32}$.

Figure 2. - Giulio Romano, Apollon écorchant Marsyas, 1525-1535.

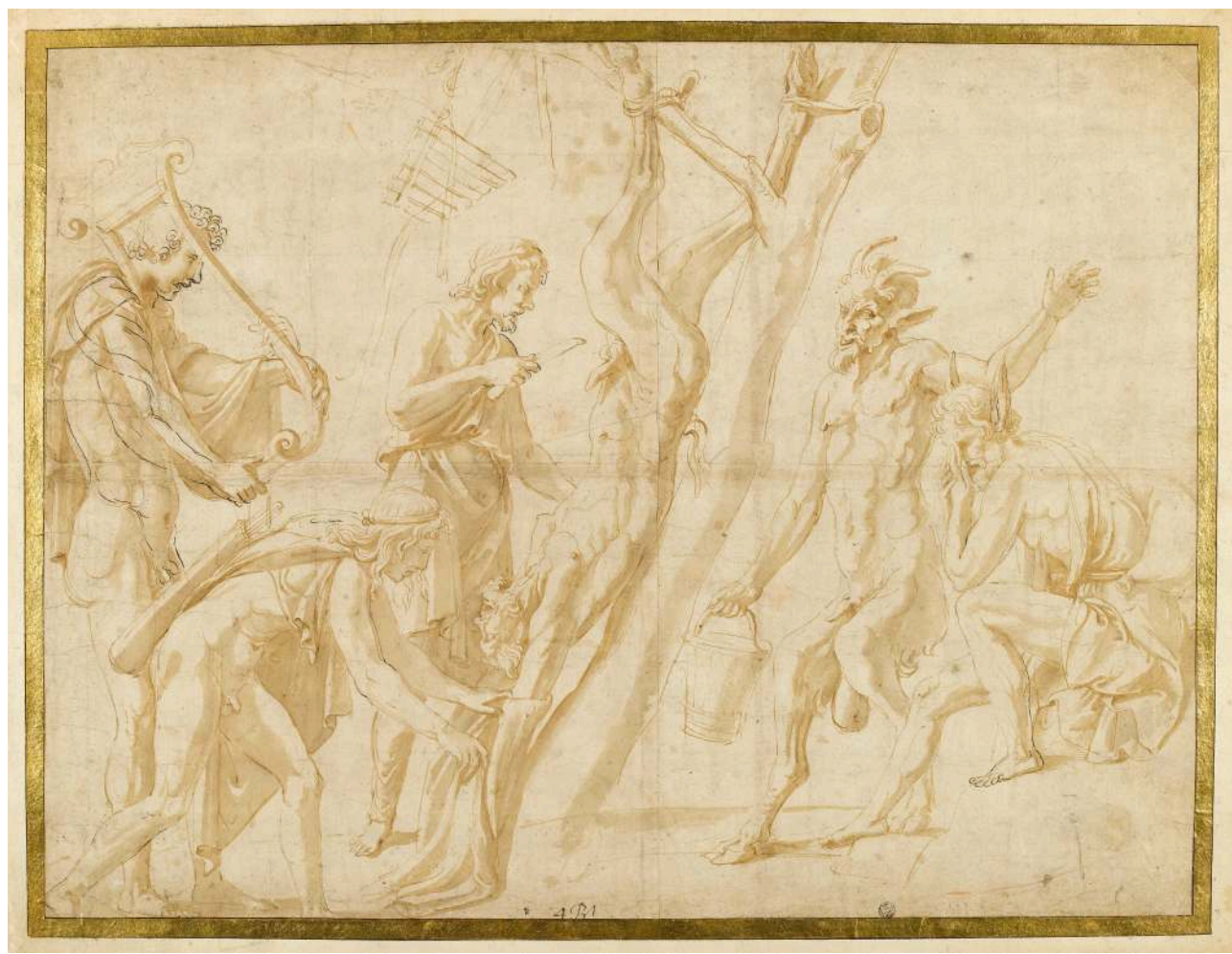

Dessin à la plume, 502 × $663 \mathrm{~mm}$

Musée du Louvre, Paris.

Le châtiment de Marsyas est une histoire de peau et en ce sens le tableau tardif paraît l'exact opposé de la Vénus d'Urbino (1538), peinte plus de trente ans auparavant, qui exposait au regard - et à la convoitise - sa carnation lumineuse. La peau de Marsyas présente au contraire une variété de teintes sombres faiblement éclairées par endroit de tons plus chauds. Le haut du corps n'a pas une teinte uniforme : plutôt claire dans la zone centrale qui entoure le nombril, la peau s'obscurcit sur le flanc droit du torse en un modelé créé par un hypothétique éclairage en provenance de la gauche. Cette ombre n'est pas non plus homogène. Elle est formée de trainées noires que l'on peut interpréter comme les traces de la putréfaction sur la peau du mourant. D'autres zones 
foncées se retrouvent sur le cou et l'épaule droite du supplicié et sous la main d'Apollon qui manie le couteau.

Du rouge s'écoule de la blessure à la poitrine causée par l'incision de la peau. Le dieu victorieux s'applique à sa tâche d'écorchement comme le chirurgien au $\mathrm{xvI}^{\mathrm{e}}$ siècle pratique la saignée en guise de traitement de la maladie infectieuse. Un deuxième personnage barbu assiste Apollon. Il participe à l'écorchement et a déjà dépouillé une large partie de la patte de Marsyas laissant paraître les muscles et le squelette dans une représentation fidèle au récit d'Ovide : "Cependant tous ses membres sont dépouillés de la peau qui les couvre. Son corps n'est qu'une plaie. Son sang coule de toutes parts. Ses nerfs sont découverts ${ }^{33}$. " Le membre écorché n'est pas sans rappeler les dessins anatomiques de plus en plus fréquents au $\mathrm{XVI}^{\mathrm{e}}$ siècle depuis la publication en 1543 à Bâle de La fabrique du corps humain du médecin André Vésale illustré de 300 planches anatomiques ${ }^{34}$. La dissection de cadavres humains donne accès à une connaissance objective du corps qui éveille l'intérêt non seulement des médecins mais également des peintres. Ces dessins d'anatomie produit par les artistes, que l'on pense à Vinci, Michelange ou Raphaël, se limitent le plus souvent cependant à des études du mouvement des membres et du corps. Il faut attendre le XvII siècle pour que la pratique anatomique devienne un genre pictural en soi dont La leçon d'anatomie de Rembrandt (1632) est l'exemple le plus connu. L'écorchement de Marsyas, en particulier le détail de la patte du satyre, fait partie des premières manifestations de ce genre en peinture ${ }^{35}$. Jacopo Palma il Giovane, émule de Titien, dont le témoignage est rapporté par Marco Boschini dans Le ricche minere della pittura veneziana (1674) avait déjà utilisé l'image du chirurgien qui, à l'aide de son bistouri, traite la partie malade, pour décrire le travail d'épuration de son maître : «[...] e scoprendo alcuna cosa, che non concordasse al delicato suo intendimento, come chirurgo benefico medicava l'infermo, se faceva di bisogno spolpargli qualche gonfiezza, ò soprabonsanza di carne [...] $]^{36}$.»

11 Un deuxième satyre à la droite du tableau transporte un seau, comme dans la composition de Romano. La fonction de ce personnage parait à première vue énigmatique. On peut imaginer que son seau sert à recueillir le sang de Marsyas, mais plus encore à nettoyer le sol des matières organiques contaminées. Cette hypothèse l'apparenterait alors aux pizzigamorti, ces vidangeurs de la peste qui ramassaient les corps et les biens infectés. Durant les périodes de peste, les services publics de Venise, à l'instar des services de santé des autres villes européennes, embauchaient des employés temporaires pour brûler les cadavres dans les sites d'incinération, transporter les malades dans les hôpitaux, nettoyer leurs biens et fumiger les maisons infectées ${ }^{37}$. Leur fonction d'assistance aux malades, mais aussi de gérants de la mort en faisaient des personnages ambigus à la fois respectés et redoutés.

Dans un article consacré à ces «bêtes de l'incinération " (beasts of burial), Jane Stevens Crawshaw relève les métaphores animales utilisées par les contemporains pour parler de cette classe de travailleurs souvent issus de milieux pauvres, parfois même criminels. On les apparente aux oiseaux charognards, aux lions et aux loups. Leur fréquentation de la mort, et par conséquent des sources d'infection, les rend menaçants aux yeux des habitants de Venise ${ }^{38}$. Ils sont perçus comme vicieux et enclins aux péchés. On les soupçonne d'abandonner les malades sur les charniers, de roder comme des bêtes la nuit dans les quartiers de la ville et de propager la maladie et la mort. Les pizzigamorti représentent l'antithèse de l'idéal de sépulture chrétienne ${ }^{39}$. Les feux qu'ils allument pour brûler les cadavres font paraître réelles les flammes de l'enfer. 
Le satyre au seau saisi en plein mouvement contribue fortement au sentiment de terreur que suscite le tableau et à l'agitation d'ensemble. Il a la bouche ouverte et ses yeux obliques au-dessus de pommettes saillantes lui donnent un regard terrifiant. Formellement, il est le double inversé de Marsyas. Sa peau foncée est dans les mêmes tons que celle de Marsyas. Sa jambe droite, partiellement cachée par le corps du roi Midas, est noire et elle aussi recouverte de fourrure animale. Contrairement à Marsyas, le bas du visage de ce satyre est couvert de poils. De petites oreilles qui ressemblent à des cornes prennent racine sur le dessus de sa tête à la limite du front et renforcent le caractère diabolique du personnage. Tout le côté gauche de la tête est sombre et se fond avec l'ombre de la barbe qui découpe un triangle sur l'épaule gauche et le haut du torse. Son bras gauche élevé et à demi-plié esquisse un geste qui fait écho à celui d'Apollon avec son couteau. Sa nature double, à la fois humain et animal, tout comme celle de Marsyas, est un autre trait du diable reconnu pour son pouvoir de se métamorphoser. Mais le mouvement qui anime ce deuxième satyre contraste avec la passivité de Marsyas.

\section{La couleur noire}

Si Satan est le prince des ténèbres, le tableau tardif de Titien peut, lui, être rapproché du courant ténébriste qui gagne la peinture à travers l'Europe dès la fin du Xvi siècle avec des peintres tels que Tintoret, Caravage, Ribera et Honthorst ${ }^{40}$. Dans cette peinture, l'obscurité ne cherche pas à reproduire l'absence de lumière du jour, elle plonge plutôt le tableau dans un moment indéfinissable ${ }^{41}$, dans une sorte de suspension du temps et de la narration.

Dans Le supplice de Marsyas, les plages sombres du tableau ont des degrés divers d'intensité. Il y a tout d'abord le noir opaque, impénétrable, des pattes velues du satyre auxquelles seules quelques touches de blanc viennent donner un peu de relief. Le chapeau noir et la tunique de l'assistant d'Apollon, la patte apparente du satyre cornu et l'ombre portée aux pieds d'Apollon présentent une même opacité qui tend à aplanir la surface. Puis, il y a l'espace dans lequel se joue la scène. Un espace dense, agité qui évoque une tempête, où le vert sombre côtoie le marron, le gris, l'ocre, le noir pur et encore le blanc. Ces teintes propres à la dernière période de l'œuvre de Titien correspondent à peu de choses près à des variations chromatiques associées aux quatre étapes du processus alchimique : nigredo, albedo, citrinnitas et rubero (noir, blanc, jaune, rouge $)^{42}$. Contrairement aux plages noires opaques sans profondeur, le fond duquel se détachent les figures est saturé de couleurs dans une sorte d'horror vacui qui exprime davantage le chaos qu'il ne définit de figures identifiables ${ }^{43}$. On distingue bien ici et là le feuillage des arbres ou le sol terreux, mais l'essentiel de la surface est couvert de taches brunâtres contrastées de coups de pinceau de peinture blanche. Les personnages euxmêmes sont peints dans ces mêmes teintes, notamment la peau de Marsyas qui occupe tout le centre de la toile, au point où on peut la qualifier, à la suite d'Itay Sapir de chair ténébreuse ${ }^{44}$. Des accents de pourpre dans les traînées de sang, dans la tunique du roi Midas, les guêtres d'Apollon, les rubans qui lient les pattes de Marsyas et la robe du musicien peuvent être interprétés, par ailleurs, selon une autre acception du mot " pourpre ", courant à l'époque de la peste, qui fait référence à une éruption cutanée de couleur rouge. Les couleurs terreuses qui priment sur la toile sont cernées de zones d'ombre que Titien vient contraster par l'application de taches de peinture blanche, 
typiques de sa pittura di macchia ${ }^{45}$ déjà soulignée par Vasari qui distinguait la première manière de Titien, minutieuse et appliquée, des taches apparentes largement brossées des derniers tableaux ${ }^{46}$. La juxtaposition de ces deux couleurs opposées dynamise le tableau tout en contribuant à son caractère dramatique.

\section{La punition divine}

La fable d'Ovide, faut-il le rappeler, est l'histoire d'une punition. Marsyas est puni d'avoir osé défier Apollon dans un concours opposant la modeste flûte du satyre à la noble lyre du dieu de l'olympe. La punition est implacable : être écorché vivant ou comme le dit Ovide "être dépouillé " de soi-même. Dans son Traicté de la Peste..., Ambroise Paré consacre le deuxième chapitre aux causes divines de la peste : «C'est une chose résolue entre les vrais Chrétiens, affirme le médecin, auxquels L'Éternel a révélé les secrets de la sagesse, que la peste et autres maladies qui adviennent ordinairement aux hommes, procèdent de la main de Dieu, ainsi que le Prophète nous enseigne ${ }^{47}$. » En réponse aux péchés des Hommes, à leur transgression de la Loi divine, le Ciel envoie le fléau de la peste et la contagion.

17 Comme le veut son châtiment, le Marsyas de Titien est bien un mort-vivant. Il a les yeux ouverts, mais sa tête légèrement orientée vers la gauche n'en laisse voir qu'un seul presque parfaitement aligné avec le nombril ${ }^{48}$ qui forme, en quelque sorte, l'œil du tableau, et peut-être même un punctum pour reprendre l'expression de Roland Barthes ${ }^{49}$, puisqu'il vise directement le spectateur. Il est dédoublé par une deuxième cavité sur la peau, peut-être une blessure si on interprète comme une traînée de sang la trace rougeâtre au milieu du torse. Malgré la délicate touche de blanc au centre de la pupille de Marsyas qui la fait luire comme celle du pesteux ${ }^{50}$, la lumière semble se retirer de l'œil du satyre en même temps que la vie. Contrairement au dessin de Romano où Marsyas hurle sa douleur dans une ultime révolte, le tableau de Titien capte le moment où le supplicié abandonne son corps à la mort.

Jodi Cranston trace un parallèle entre la composition du Supplice de Marsyas formée d'un personnage central flanqué de chaque côté de personnages secondaires de l'organisation formelle de la sacra conversazione, typique de la peinture religieuse $\mathrm{du} \mathrm{xv}^{\mathrm{e}}$ siècle $^{51}$. Au lieu de la figure du saint ou de la vierge, Titien place au centre de la toile, dans une sorte de "mythologie sacrée ${ }^{52}$ ", le corps inversé du satyre dont l'animalité et la noirceur l'associent davantage au diable qu'au Christ. Le positionnement en croix du corps de Marsyas rappelle aussi les scènes de crucifixion, particulièrement celle de saint Pierre dans la fresque La crucifixion de saint Pierre (1426) de Masaccio, mais en détourne le sens en remplaçant la figure du saint par un personnage d'apparence antéchristique. Par ailleurs, la flûte, elle aussi suspendue, et la branche pendante de l'arbre forment avec le corps de Marsyas un trio qui rappelle le Christ et les deux larrons.

\section{L'humeur mélancolique}

19 Parmi les causes de la peste, la peur elle-même, aux dires des médecins de l'époque, est désignée responsable de la contagion. Ludovico Antonio Muratori (1672-1750) pointera explicitement l'abattement moral et la mélancolie comme terreaux de l'infection: «L'appréhension, écrit-il, la terreur et la mélancolie sont, elles aussi, une peste, car 
elles abattent notre optimisme et disposent la masse des humeurs à recevoir facilement et d'une certaine façon à attirer de loin le poison qui règne, ainsi que l'expérience l'a montré en une infinité de $\operatorname{cas}^{53}$. » Ambroise Paré va jusqu'à recommander en mesure de prévention de: «[...] se tenir joyeux, en bonne et petite compagnie, et parfois ouïr chantres et instruments de musique, et aucunes fois lire et ouïr quelques lectures plaisantes $[. . .]^{54}$.»

La notion de mélancolie, troisième figure du malheur à la Renaissance selon Azouvi, renvoie à la théorie des quatre humeurs. Le mot vient du grec melagkolia qui signifie "bile noire ». C'est, dans l'équilibre des humeurs, l'excès de cette bile, dite aussi atrabile, qui ferait sombrer dans la mélancolie $e^{55}$. En avant-propos de Saturne et la mélancolie, Raymond Klibansky insiste sur l'importance à travers l'histoire du symbolisme de la couleur noire propre à la mélancolie : «Si l'on examine l'histoire du terme mélancolie ou bile noire dans les écrits de l'Antiquité grecque, on aurait tort de négliger une composante pré-scientifique, l'importance de la couleur noire et tout ce qu'elle évoque à partir d'Homère ${ }^{56}$." Dans la théorie des humeurs, l'humeur mélancolique est aussi associée à la terre pour sa sécheresse et sa concentration. Elle aurait pour effet de retirer l'âme du dehors vers le dedans du corps et d'évoquer métaphoriquement la concentration intellectuelle et l'introversion: "Le studioso est donc bien un mélancolique, puisqu'il réalise en lui-même cette astringence qui caractérise la terre, puisqu'il tend toujours vers son propre centre ${ }^{57}$.» La notion d'humeur, tel qu'entendue à la Renaissance, a ceci de particulier qu'elle définit une substance corporelle, mais qui produit un effet sur l'âme. Ainsi, elle crée un pont entre la matière et l'esprit, ces deux entités opposées de la philosophie classique, à la façon d'une sorte de neurotransmetteur avant la lettre. Pour l'homme de la Renaissance, la mélancolie serait la peste de l'âme.

21 La mélancolie a aussi pour caractéristique sa plasticité ce qui l'apparente à l'œuvre d'art. Le mélancolique est réputé pour prendre toutes les formes, comme le diable, sous l'influence de la bile noire dont les vapeurs montent au cerveau et entraînent des hallucinations, des méprises - ce qui explique le choix de cette figure pour représenter le manque de jugement du roi - et des déformations ${ }^{58}$. La tristesse ouvrait une brèche dans l'âme du mélancolique par laquelle le diable pouvait plus facilement s'infiltrer ${ }^{59}$. La mélancolie est associée à l'automne, au froid, au vent du nord, à la couleur noire, au grand âge et au pendu ${ }^{60}$. La mélancolie serait aussi une conséquence de la peste à travers le délire qu'elle provoque.

Dans Le supplice de Marsyas de Titien, un personnage assis à droite, la tête appuyée sur sa main, adopte la pose caractéristique du mélancolique dont les traits ont été fixés par Dürer dans la gravure Melencolia I datée de $1517^{61}$. Inspiré également du dessin de Giulio Romano, l'homme âgé représente le roi Midas qui, dans les Métamorphoses d'Ovide, aurait servi d'arbitre lors du concours opposant Apollon et le dieu Pan. Son erreur de jugement en faveur du dieu Pan lui aurait valu des oreilles d'âne. Dans le tableau de Titien, c'est la vue du spectacle qui se déroule sous ses yeux qui semble plonger le roi dans la mélancolie. En même temps, son regard est dirigé vers le sol et son visage partiellement caché par sa main place le personnage intérieurement en retrait de la scène, dans une sorte d'état méditatif caractéristique de l'humeur mélancolique. Sa passivité l'oppose à l'action du satyre derrière lui et à celle d'Apollon dans la partie gauche du tableau, mais l'apparente à Marsyas qui subit son sort aussi résigné qu'un martyr chrétien. 

toutefois s'entendre sur l'interprétation à donner de cette représentation de l'artiste par lui-même dans le rôle d'un personnage mythologique au jugement défaillant. D'autres avant et après le peintre vénitien ont été tenté par l'autoportrait dissimulé dans l'œuvre. On pense à Michel-Ange qui se représente littéralement " dans la peau » de saint Barthélemy, un autre dépiauté célèbre, dans la fresque du Jugement dernier ou aux nombreux autoportraits de Caravage qui jalonnent son œuvre. Ce rôle de témoin adopté par l'artiste introduit dans le tableau une subjectivité que Jodi Cranston qualifie d'antihéroïque propre à la sensibilité des débuts de la modernité6 ${ }^{63}$. Elle annonce également une position d'observateur de l'artiste en retrait de l'action. La mélancolie $\mathrm{du}$ roi-artiste pourrait traduire l'expression par l'art de la douleur physique, mais surtout morale, qui serait celle de Titien, en ces temps d'apocalypse. Par ailleurs, Apollon, qu'il soit chirurgien ou sacrificateur, son assistant et le satyre au seau sont visiblement des êtres d'action représentés en mouvement. Il y a là, pourrait-on dire, une interrogation sur le pouvoir de l'artiste d'agir sur le réel qui cède la place à l'homme de science, et même à l'homme de maintenance, responsables de la survie des corps.

\section{La putréfaction et l'informe}

Si la couleur noire est commune à la peste, au diable et à la mélancolie, ces trois formes de l'horreur sont également impliquées à divers degrés dans le processus de la putréfaction. François Azouvi dira que : «[...] la mélancolie, le diable et la peste sont trois modalités de l'infect, du corrompu, du putride [...] ${ }^{64}$.» La peste est associée aux eaux croupissantes, aux vapeurs malsaines, aux lieux reclus. Azouvi écrit que le diable fréquente les cimetières, les fosses d'aisance et on dit de lui qu'il dégage une " odeur de charogne ${ }^{65}$ ». L'humeur mélancolique serait elle aussi une pourriture qui part de la rate et se propage au cerveau. Dans les trois cas, la putréfaction engendre une altération : le sang qui s'écoule du pestiféré devient noir en séchant, le diable se consume dans les chaleurs de l'enfer et la bile noire fait perdre peu à peu au mélancolique l'usage de la raison. La putréfaction, bien qu'associée à la mort, est un processus, qui, loin d'être figé, se poursuit dans le temps. Le putride est affaire de matière. Il s'attaque au corps et ramène l'Homme à son essence première.

La matière est aussi le souci du peintre qui a pour principal moyen d'expression des pigments colorés. L'intérêt de Titien pour la matière picturale n'est plus à démontrer depuis la publication de Marco Boschini en 1674 sur la peinture vénitienne ${ }^{66}$. Non seulement Titien est réputé pour peindre parfois directement avec les mains dans les dernières étapes du processus de création, ce qui traduit le caractère artisanal de sa pratique, mais son coup de pinceau apparent insiste sur la matérialité de l'œuvre au détriment de son pouvoir de représentation. David Rosand a bien décrit la grande variation dans l'application de la peinture dans Le supplice de Marsyas, du trait large et flou de l'arrière-plan et de l'environnement naturel à l'exécution fine de certains objets, particulièrement métalliques, comme le couteau d'Apollon et la couronne de Midas. Une diversité qui vient animer la surface du tableau et lui insuffler une vie qui contraste avec le caractère tragique du sujet ${ }^{67}$.

Lorsqu'appliquée à larges traits, la peinture tend à dissoudre les formes qui se mêlent les unes aux autres. Dans l'œuvre tardive de Titien, la technique picturale conduit à

Cahiers d'études italiennes, 31 | 2020 
l'informe, mais Le supplice de Marsyas en représente l'expression la plus poussée. Ainsi, chez Titien : "[...] l'informe est en soi un objet esthétique $[. . .]^{68}$.» C'est le cas tout spécialement du fond du tableau qu'on ne peut décrire autrement qu'en termes de coups de pinceau, si bien qu'aucune profondeur illusionniste ne se révèle. Au contraire, le fond tend à rogner les figures comme les bacilles de la peste sous la peau des vivants et des morts.

Dans la toile de Titien, le corps de Marsyas occupe la plus grande part de l'espace. Ses dimensions monumentales - il faut se rappeler que le tableau mesure $212 \mathrm{~cm}$ de haut par $207 \mathrm{~cm}$ de large - exercent une présence oppressive sur le spectateur. La composition en rappelle une autre plus tardive qui elle aussi, et peut-être encore plus directement, représente le corps dans toute sa matérialité. Il s'agit du Bœuf écorché (fig. 3) de Rembrandt, notamment sa version du Louvre datée de 1655. Dans les deux œuvres, le corps de la bête - dans le cas de Marsyas, la bête est à moitié humaine - est suspendu librement par les pieds, pattes écartées. Le ventre éclairé se détache d'un environnement sombre et constitue le centre du tableau. Rembrandt, comme Titien avant lui, adopte une gestuelle brute qui rend apparente la matérialité de la peinture. Pour Mieke Bal, le choix de cette technique dans Le bøuf écorché a pour conséquence de rendre métaphoriquement par l'odeur de la peinture, la puanteur de la chair morte ${ }^{69}$.

Figure 3. - Rembrandt, Le bœuf écorché, 1655.

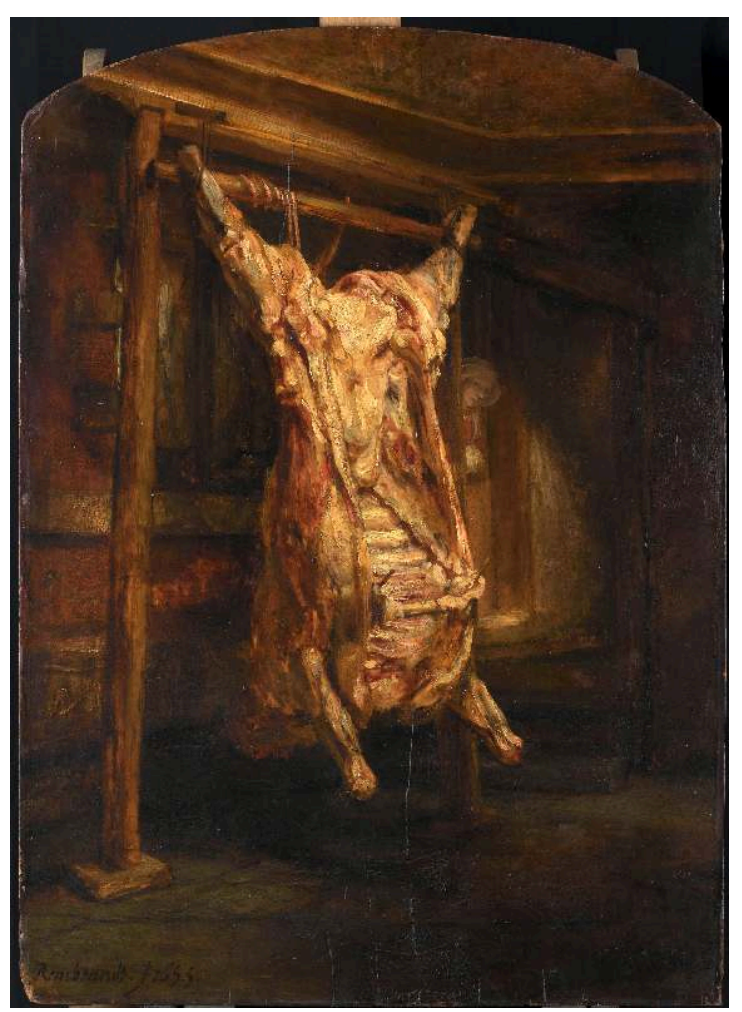

Huile sur bois, $94 \times 69 \mathrm{~cm}$.

Musée du Louvre, Paris.

Mais si Le bouf écorché représente dans son interprétation la plus littérale une pièce de viande, Le supplice de Marsyas réfère explicitement à un sacrifice. Elaine Scarry, citée par Mieke $\mathrm{Bal}^{70}$, définit le rituel du sacrifice comme le retournement de l'intérieur vers l'extérieur ${ }^{71}$. L'enveloppe du corps, la peau, est fissurée, et les contours stables de son 
identité sont menacés. La forme de la mort (the shape of death) selon Bal serait l'ouverture du corps.

\section{Le dérèglement}

29 L'inversion de la figure de Marsyas coïncide avec le renversement des valeurs lumineuses de la peinture classique. La masse noire des pattes du satyre et le tronc d'arbre auquel elles sont pendues, assombrit le haut du tableau tandis que la peau du torse, des bras et du visage est éclairée, ce qui contrevient à l'organisation traditionnelle de l'espace - on pense par exemple au Jugement dernier de Michelange où les ténèbres, l'enfer, la fange occupent la partie inférieure du tableau dominée par la lumière céleste et la présence divine. Cette inversion est redoublée par la position des personnages: le dieu agenouillé près du corps de sa victime dont l'instrument de musique, la flûte de Pan, trône dans le haut du tableau. Par ailleurs, le corps de Marsyas, jambes écartées et bras ballants à l'intérieur d'une surface pratiquement carrée, reproduit à peu de choses près la figure de l'Homme vitruvien de Vinci ${ }^{72}$, mais en l'inversant. Les dimensions du corps de Marsyas contreviennent également aux proportions harmonieuses de la composition selon Alberti. Comparativement aux autres personnages, sa masse, particulièrement son torse, paraît démesurée $e^{73}$. Ceci a pour effet de produire un espace confiné exacerbé par l'absence de perspective qui empêche l'œil du spectateur d'échapper à l'horreur de la scène. Ici, il n'y a pas de rédemption possible.

L'idée d'un monde sens dessus-dessous correspond étrangement au désordre provoqué par la peste. L'épidémie entraîne la désorganisation sociale, l'abandon des rites, notamment funéraires, et de la distinction des classes sociales qui se retrouvent égales devant la peur, la souffrance, la mort et le deuil :

La peste est, sans aucun doute, entre toutes les calamités de cette vie, la plus cruelle et véritablement la plus atroce. C'est à grande raison qu'on l'appelle par antonomase le Mal. Car il n'y a sur terre aucun mal qui soit comparable à la peste. Dès que s'allume dans un royaume ou une république ce feu violent et impétueux, on voit les magistrats abasourdis, les populations épouvantées, le gouvernement politique désarticulé. La justice n'est plus obéie ; les métiers s'arrêtent; les familles perdent leur cohérence, et les rues leur animation. Tout est réduit à une extrême confusion $^{74}$.

31 Dans cette vision de fin du monde peinte par Titien, la figure pensive du roi Midas contraste avec le prosaïsme des autres personnages, particulièrement Apollon, dont le regard rivé sur le corps de sa victime (ou de son patient) guide son instrument, ou son assistant appliqué à son travail de dissection, mais aussi le satyre équipé quant à lui de son seau, empressé de nettoyer les lieux du sang et des humeurs contaminées du mourant.

Comme toutes les œuvres complexes, Le supplice de Marsyas ne peut se cantonner à une lecture univoque. Le tableau, par ses ambiguïtés, résiste à une lecture totalisante et définitive comme l'a bien démontré Jutta Held dans son "analyse des analyses ${ }^{75}$ ». Celle-ci note judicieusement que le savoir factuel sur l'œuvre, depuis sa redécouverte en 1924, n'a progressé que de façon marginale comparativement à l'abondance d'interprétations auxquelles elle a donné lieu. Chaque interprétation doit contourner ses contradictions ou au mieux assumer ses paradoxes pour offrir une argumentation cohérente et signifiante pour un lecteur d'aujourd'hui. Titien, lui-même, comme le 
prouvent les nombreux pentimenti, a sans cesse corrigé son langage pictural, ce qui peut avoir eu comme effet d'en accentuer les contradictions, sans compter les interventions parfois malheureuses de ses assistants d'atelier ${ }^{76}$. Malgré tout, on peut s'étonner que la présence foudroyante de la peste à Venise au moment de la création du tableau ait aussi peu attiré l'attention de la critique au sujet d'une œuvre qui représente pourtant et avec insistance un mourant. Tout porte à croire que c'est précisément l'ampleur du phénomène et ses liens étroits avec la vie de Titien qui ont provoqué cette sorte d'aveuglement.

\section{BIBLIOGRAPHIE}

AIKEMA Bernard, « Tiziano, la maniera e il pubblico », Mitteilungen des Kunsthistorischen Institutes in Florenz, vol. 39, 1995, p. 167-184.

Azouvi François, « La peste, la mélancolie et le diable ou l'imaginaire réglé », Diogane, $\mathrm{n}^{\circ}$ 108, oct. 1979, p. 124-143.

AlAZARD Jean, La Venise de la Renaissance, [Paris], Hachette, 1956.

BAL Mieke, Reading Rembrandt. Beyond the Word-Image Opposition, Amsterdam University Press, 2006 (1991).

BARTHES Roland, La Chambre claire. Note sur la photographie, Paris, Éditions Cahiers du cinéma, Gallimard, Seuil, 1980.

BatAille Georges et Strauss Jonathan, « Death and Sacrifice », Yale French Studies, nº 78, 1990, p. 9-28.

BEAUsSANT Philippe, Titien. Le chant du cygne, Paris, Fayard, 2009.

BÉGUIN Sylvie, Titien, Paris, Flammarion, coll. « Les classiques de l'art », 2006.

BoschinI Marco, Le Minere della pittura, compendiosa informazione di Marco Boschini, non solo delle pitture publiche di Venezia, ma dell' isole ancora circonvicine, Venise, F. Nicolini, 1674 (1664), 7 parties en 1 vol.

CAMPBELl J. Stephen, « Bronzino's Fable of Marsyas: Anatomy as Myth », dans Victor I. Stoichita (éd.), Le corps transparent, Rome, L'Erma di Bretschneider, 2013, p. 173-194.

CASSEGRAIN Guillaume, «Titien. Actualité des études », Perspective. Actualité en histoire de l'art, $\mathrm{n}^{\circ} 2$, 2008, p. 205-226.

CRANSTON Jodi, The Muddied Mirror, Materiality and Figuration in Titian's Later Paintings, University Park, Pennsylvania State University Press, 2010.

DeLUMEAU Jean, La peste en Occident. Une ville assiégée : XIV -XVIII siècles, Paris, Fayard, 1978.

GeNTILI Augusto, Titien, traduit de l'italien par Anne Guglielmetti, Arles, Actes Sud, 2012.

HELD Jutta, «Titian's Flaying of Marsyas: An Analysis of the Analyses », Orford Art Journal, vol. 31, $\mathrm{n}^{\circ}$ 2, 2008, p. 181-194. 
Holly Michael Ann, The Melancholy Art, Princeton University Press, 2013.

KLIBANSKY Raymond, PANOFSKY Erwin et SAXL Friz, Saturne et la mélancolie. Études historiques et philosophiques : nature, religion, médecine et art, traduit de l'anglais et d'autres langues par Fabienne Durand-Bogaert et Louis Évrard, Paris, Gallimard, 1989 (1964).

McNeILl William H., Le temps de la peste. Essai sur les épidémies dans l'histoire, Paris, Hachette, 1978 (1976).

MURATORI Ludovico Antonio, Del governo della peste, e della maniere di guardarsene, Modène,

B. Soliani, 1714

NAPHY William et SPICER Andrew, La peste noire, 1345-1730. Grandes peurs et épidémies, traduit de l'anglais par Arlette Sancery, suivi de « Sur quelques origines » d'Emmanuel Le Roy Ladurie, Paris, Éditions Autrement, coll. « Mémoires », 2003.

NoHL Johannes, La mort noire. Chronique de la peste, traduit de l'anglais par Anne Howe, édition présentée par Jacqueline Brossolet, Paris, Payot, 1986.

OviDE, Métamorphoses, livre VI, traduit et annoté par A.-M. Boxus et J. Poucet, Bruxelles, Biblioteca selecta, 2006. Disponible en ligne sur <http://bcs.fltr.ucl.ac.be/META/00.htm> (consulté le 25 juillet 2020).

PANOFSKY Erwin, Titien. Questions d'iconographie, traduit de l'anglais par Éric Hazan, s. l., Hazan, 2009 (1969).

PARÉ Ambroise, Traicté de la Peste, de la petite Verolle \& Rougeolle : avec une brefve description de la Lepre, Paris, Imprimerie André Wechel, 1568.

"Paré Ambroise », Encyclopédie de l’Agora, en ligne sur <http://agora.qc.ca/dossiers/ Ambroise_Pare> (consulté le 26 juillet 2020).

« La peste », Larousse. Encyclopédie en ligne, <www.larousse.fr/encyclopedie/divers/peste/78778> (consulté le 26 juillet 2020).

PIETERS Corinne, «L'anatomie entre art et science au XVI siècle : autopsie d'un regard », Communication et langages, $\mathrm{n}^{\circ} 127,2002, \mathrm{p} .61-77$

REARICK W. R., « Titian's Later Mythologies », Artibus et Historiae, vol. 17, n 33, 1996, p. 23-67.

RomAni Vittoria, Titien et la fin de la Renaissance à Venise. Jacopo Bassano, Le Tintoret, Paul Véronèse, Paris, Le Figaro, coll. « Les grands maîtres de l'art », 2008.

Rosand David, « “Most Musical of Mourners, Weep Again!”: Titian's Triumph of Marsyas », Arion: A Journal of Humanities and the Classics, $3^{\mathrm{e}}$ série, vol. 17, $\mathrm{n}^{\circ}$ 3, hiver 2010, p. 17-43.

RZEPINSKA Maria et MALCHAREK Krystyna, « Tenebrism in Baroque Painting and Its Ideological Background », Artibus et Historiae, vol. 7, n 13, 1986, p. 91-112.

SANTA MARIA Francisco de, Historia das sagradas congregacoes dos conegos seculares de S. Jorge em alga de Venesa e de S. Joaõ evangelista em Portugal, Lisbonne, 1671.

SAPIR Itay, Ténèbres sans leçons. Esthétique et épistémologie de la peinture ténébriste romaine, 1595-1610, Berne, Berlin, Bruxelles, Francfort-sur-le-Main, New York, Oxford et Vienne, Peter Lang, 2012.

SCARRY Elaine, The Body in Pain: The Making and Unmaking of the World, Oxford et New York, Oxford University Press.

SoNTAG Susan, Devant la douleur des autres, s. 1., Christian Bourgeois éditeur, 2003 (2002). 
Stevens CRawshaw Jane L., « The Beasts of Burials: Pizzigamorti and the Public Health for the Plague in Early Modern Venice ", Social History of Medecine, vol. 24, n 3, 2011, p. 570-577.

VASARI Giorgio, Les vies des meilleurs peintres, sculpteurs et architectes, traduction et édition commentée sous la direction d'André Chastel, Arles, Actes Sud, 2005, 2 vol.

ZANETTE Laura, « Tre predicatori e la peste: 1575-1577 », Lettere italiane, vol. 42, n 3, juillet 1990, p. 430.

\section{NOTES}

1.W. H. McNeill, Le temps de la peste. Essai sur les épidémies dans l'histoire, traduit de l'américain par C. Yelnick, s. 1., Hachette, 1976, p. 130.

2. W. Naphy et A. Spicer, La peste noire, 1345-1730. Grandes peurs et épidémies, traduit de l'anglais par A. Sancery, suivi de "Sur quelques origines » de E. Le Roy Ladurie, Paris, Éditions Autrement, 2003, p. 70-92.

3. J. Nohl, La mort noire. Chronique de la peste, Paris, Payot, 1986, p. 122.

4. J. L. Stevens Crawshaw, "The Beasts of Burials: Pizzigamorti and the Public Health for the Plague in Early Modern Venice ", Social History of Medecine, vol. 24, n 3, 2011, p. 570.

5. Si l'année de naissance de Titien fait l'objet de débats et de discussion (Augusto Gentili dans son Titien de 2012 la situe entre 1483 et 1485 alors qu'André Chastel dans l'édition critique de 2005 des Vies de Vasari propose plutôt 1488-1489), la cause de la mort du peintre, peste ou vieillesse, ne fait pas non plus l'unanimité des biographes.

6. Traduction de l'auteure: «[...] nous avons vu durant l'horrible peste de cette année, [le Lazaretto Vecchio] rempli de huit mille personnes infectées, avec grand dommage et douleur dans la cité affligée [...]. » (F. Sansovino, Venetia citta nobilissima e singolare, livre V, Venise, 1581, p. 84-85)

7. E. Panofsky, Titien. Questions d'iconographie, s. l., Hazan, 2009, p. 302.

8. J. Held, «Titian's Flaying of Marsyas: An Analysis of the Analyses », Oxford Art Journal, vol. 31, $\mathrm{n}^{\circ} 2,2008$, p. 183.

9. Certains auteurs prétendent que le format du tableau original aurait été modifié et réduit de chaque côté. Voir J. Held, « Titian's Flaying of Marsyas » art. cité, p. 183-184.

10. W. R. Rearick, « Titian's Later Mythologies », Artibus et Historiae, vol. 17, n 33, 1996, p. 62.

11. Ibid.

12. D. Rosand, « "Most Musical of Mourners, Weep Again!”: Titian's Triumph of Marsyas ", Arion: A Journal of Humanities and the Classics, $3^{\mathrm{e}}$ série, vol. 17, $\mathrm{n}^{\circ}$ 3, hiver 2010, p. 25.

13. A. Gentili, Titien, trad. de l'italien par A. Guglielmetti, Arles, Actes Sud, 2012, p. 374.

14. D. Rosand, « Most Musical of Mourners », art. cité, p. 19.

15. E. Panofsky règle le sort du tableau dans une note de bas de page à la fin de Titien, ouvr. cité, p. 302.

16. Voir la méta-analyse de J. Held, « Titian’s Flaying of Marsyas », art. cité, p. 181-194.

17. W. H. McNeill, Le temps de la peste, ouvr. cité, p. 165.

18. J. Held, « Titian's Flaying of Marsyas », art. cité, p. 183.

19. S. Sontag, Devant la douleur des autres, s. 1., Christian Bourgeois éditeur, 2003, p. 69.

20. E. Panofsky, ouvr. cité, p. 302.

21. J. Cranston, The Muddied Mirror. Materiality and Figuration in Titian's Later Paintings, University Park, The Pennylvania State University Press, 2009, p. 50.

22. J. Delumeau, La peur en Occident. Une citée assiégée : XVI'- XVIII siècles, Paris, Fayard, 1978, p. 103. 
23. F. Azouvi, «La peste, la mélancolie et le diable, ou l'imaginaire réglé », Diogane, ${ }^{\circ} 108$, oct. 1979, p. 124-143.

24. "Paré Ambroise», Encyclopédie de l'Agora, en ligne sur <http://agora.qc.ca/dossiers/ Ambroise_Pare> (consulté le 26 juillet 2020).

25. A. Paré, Traicté de la Peste, de la petite Verolle \& Rougeolle : avec une brefve description de la Lepre, Paris, Imprimerie André Wechel, 1568, p. 1. Dans sa préface, Paré nous apprend qu'il a non seulement soigné de nombreux pestiférés, mais qu'il a lui-même été atteint par la maladie.

26. «La peste", Larousse. Encyclopédie en ligne, <www.larousse.fr/encyclopedie/divers/peste/ 78778> (consulté le 26 juillet 2020).

27. F. Azouvi, « La peste, la mélancolie et le diable », ouvr. cité, p. 129.

28. J. Delumeau, La peur en Occident, ouvr. cité, p. 105-106.

29. D. Rosand avance que Titien possédait probablement un dessin de ce projet qui lui aurait été donné par l'artiste dont il avait fait le portrait (D. Rosand, « Most Musical of Mourners », art. cité, p. 19).

30. J. Cranston, The Muddied Mirror, ouvr. cité, p. 53.

31. J. Held, « Titian's Flaying of Marsyas ", art. cité, p. 187.

32. Ovide, Métamorphoses, traduit et annoté par A.-M. Boxus et J. Poucet, Bruxelles, Biblioteca selecta, 2006, livre VI, lignes 382-400. Disponible en ligne sur <http://bcs.fltr.ucl.ac.be/META/ 00.htm> (consulté le 25 juillet 2020).

33. Ibid.

34. C. Pieters, "L'anatomie entre art et science au $\mathrm{xvI}^{\mathrm{e}}$ siècle: autopsie d'un regard", Communications et langages, $\mathrm{n}^{\circ} 127,2001, \mathrm{p} .66$.

35. Bronzino, dans une peinture sur le même sujet du musée de l'Ermitage datée de 1531-1532, montre Marsyas étendu au sol, la jambe déjà « pelée » comme un fruit.

36. Traduction de l'auteure : «[...] et découvrant une chose qui ne concordait pas avec sa délicate intention, comme un chirurgien bienfaisant, il corrigeait l'infirmité, la dépouillant de quelque gonflement ou d'une surabondance de chair [...].» (M. Boschini, Le ricche minere della pittura veneziana, Venise, Francesco Nicolini, 1674, p. b5)

37. J. L. Stevens Crawshaw, « The Beasts of Burials », art. cité, p. 574.

38. Ibid., p. 575.

39. Ibid., p. 584.

40. M. Rzepinska et K. Malcharek, "Tenebrism in Baroque Painting and Its Ideological Background ", Artibus et Historiae, vol. 7, no 13, 1986, p. 92.

41. Ibid.

42. Ibid., p. 104.

43. I. Sapir, Ténèbres sans leçons. Esthétique et épistémologie de la peinture ténébriste romaine, 1595-1610, Berne, Berlin, Bruxelles, Francfort-sur-le-Main, New York, Oxford et Vienne, Peter Lang, 2012, p. 51. C'est d'ailleurs l'expression qu'utilise Panovsky pour conclure que le tableau n'est probablement pas de la main de Titien (E. Panovsky, Titien, ouvr. cité, p. 302).

44. I. Sapir, Ténèbres, ouvr. cité, p. 50.

45. Ibid., p. 49.

46. G. Vasari, Les vies des meilleurs peintres, sculpteurs et architectes, traduit et commenté par A. Chastel (éd.), Arles, Actes Sud, 2005, livre X, p. 34.

47. A. Paré, Traicté de la Peste, ouvr. cité, p. 3.

48. Le nombril, faut-il le rappeler, est ce qui relie le corps de l'enfant à celui de la mère et lui permet de vivre en le nourrissant.

49. R. Barthes, La Chambre claire. Note sur la photographie, Paris, Éditions Cahiers du cinéma, Gallimard, Seuil, 1980, p. 9.

50. J. Delumeau, La peur en Occident, ouvr. cité, p. 105.

51. J. Cranston, The Muddied Mirror, ouvr. cité, p. 51. 
52. Ibid.

53. L. A. Muratori, Del governo della peste, e della maniere di guardarsene, Modène, 1714, p. 329. Cité par J. Delumeau, La peur en Occident, ouvr. cité, p. 117.

54. A. Paré, Euvres, Paris, éd. de P. Tartas, 1969 (1585), chap. 3, p. VIII.

55. F. Azouvi, « La peste, la mélancolie et le diable », ouvr. cité, p. 126.

56. R. Klibansky, E. Panofsky et F. Saxl, Saturne et la mélancolie, études historiques et philosophiques : nature, religion, médecine et art, trad. de l'anglais et d'autres langues par F. Durand-Bogaert et L. Évrard, Paris, Gallimard, 1989, p. 10.

57. F. Azouvi, « La peste, la mélancolie et le diable », ouvr. cité, p. 127.

58. Ibid., p. 139.

59. R. Klibansky, E. Panofsky et F. Saxl, Saturne et la mélancolie, ouvr. cité, p. 137.

60. M. A. Holly, The Melancholy Art, Princeton University Press, 2013, p. 138.

61. R. Klibansky, E. Panofsky et F. Saxl, Saturne et la mélancolie, ouvr. cité, p. 473.

62. J. Held, « Titian's Flaying of Marsyas », art. cité, p. 187.

63. J. Cranston, The Muddied Mirror, ouvr. cité, p. 69.

64. F. Azouvi, « La peste, la mélancolie et le diable », ouvr. cité, p. 130.

65. Ibid.

66. M. Boschini, Le Minere della pittura, ouvr. cité, p. b4-b6.

67. D. Rosand, « Most Musical of Mourners ", art. cité, p. 24.

68. I. Sapir, Ténèbres, ouvr. cité, p. 46.

69. M. Bal, Reading Rembrandt. Beyond the Word-Image Opposition, Amsterdam University Press, 2006 (1991), p. 386.

70. Ibid., p. 375.

71. E. Scarry, The Body in Pain. The Making and Unmaking of the World, New York et Oxford, Oxford University Press, p. 131.

72. D. Rosand, « Most Musical of Mourners », art. cité, p. 27.

73. J. Held, « Titian's Flaying of Marsyas », art. cité, p. 190.

74. F. de Santa Maria, Historia das sagradas congregacoes des conegos seculares de S. Jorge en alga de Venesa e de S. Joaõ evangelista em Portugal, Lisbonne, 1671, p. 270-272. Cité par J. Delumeau, ouvr. cité, p. 112.

75. J. Held, « Titian's Flaying of Marsyas », art. cité, p. 181-194.

76. Ibid., p. 192.

\section{RÉSUMÉS}

Cette relecture d'un tableau tardif de Titien s'intéresse à son contexte sociohistorique, l'épidémie de peste de San Carlo à Venise qui aurait emporté le peintre en 1576. En adoptant l'angle de la peste noire, l'auteure fait valoir l'importance de la couleur noire qui est pigment, mais aussi signe sur la peau du pestiféré, symbole du diable dans la tradition chrétienne et dominante de l'humeur mélancolique. Ainsi, la fable d'Ovide telle qu'illustrée par Titien peut se lire comme la rencontre de savoirs médicaux, de croyances et de sensibilités propres au XVI ${ }^{\mathrm{e}}$ siècle finissant.

Questa rilettura di un'opera tardiva di Tiziano s'interessa al suo contesto sociale e storico, l'epidemia di peste di San Carlo a Venezia durante la quale è morto il pittore nel 1576 . Adoperando l'angolo della peste nera, l'autrice dell'articolo dimostra l'importanza del colore 
nero che è pigmento, ma anche segno sulla pelle dell'appestato, simbolo del diavolo nella tradizione cristiana e dominante dell'umore melanconico. Così, la favola di Ovidio comme illustrata da Tiziano si può leggere come l'incontro tra conoscenze mediche, fede et sensibilità della fine del Cinquecento.

This article sheds a new light on Titian's late painting, Flaying of Marsyas, taking into account its historical and sociological context, the Plague of San Carlo in Venice which killed the artist in 1576. Through the perspective of the Black Plague (peste nera), the author shows the importance of black not only as the color but also as a sign on the skin of the infected patient, a symbol of the devil in the Christian tradition and the prevailing humor of melancholy. Thus, Ovid's fable as illustrated by Titian can be interpreted as the confluence of medical knowledge, beliefs and sensibilities of the end of the 16th century.

INDEX

Keywords : Titian, Marsyas, plague, melancholy, Venice

Parole chiave : Tiziano, Marsia, peste, melanconia, Venezia

Mots-clés : Titien, Marsyas, peste, mélancolie, Venise

\section{AUTEUR}

\section{SILVIE BERNIER}

Docteure, Université de Sherbrooke, Québec 